\title{
PASADO, PRESENTE Y FUTURO DE LA DIDÁCTICA DE LAS CIENCIAS
}

\author{
PORLÁN ARIZA, R. \\ Departamento de Didáctica de las Ciencias. Universidad de Sevilla. Avenida Ciudad Jardín, 22. \\ 41005 Sevilla. Miembro del Grupo DIE (Didáctica e Investigación Escolar) y del Proyecto Curricular IRES \\ (Investigación y Renovación Escolar).
}

\section{UNA CONCEPCIÓN COMPLEJA DE LAS DISCIPLINAS}

Uno de los debates epistemológicos más importantes de los últimos veinticinco años trata de las similitudes y diferencias que existen entre los modos de producción científica de las ciencias experimentales y de las ciencias sociales (Bunge, 1985). Este debate, aún no concluido, está presidido por un principio básico: definir los rasgos que caracterizan el conocimiento científico y que lo distinguen de otras formas de conocimiento. En el caso de la didáctica de las ciencias este debate presenta perfiles ciertamente paradójicos y problemáticos, ya que, por un lado, las ciencias experimentales son una fuente primordial para el conocimiento didáctico y constituyen las disciplinas de origen de gran parte de la comunidad investigadora implicada y, por otro, su objeto de estudio, la educación científica y la formación del profesorado de ciencias, en la medida que se desarrolla en sistemas humanos, se ubica en el ámbito de las ciencias sociales.

Con frecuencia, el contraste epistemológico entre las ciencias experimentales y las ciencias sociales ha sido presentado como un contraste entre disciplinas maduras y consolidadas (las primeras) y disciplinas jóvenes y difusas (las segundas) (Toulmin, 1972). Es cierto que la edad de una ciencia es una variable que influye poderosamente en el rigor de sus métodos y en la racionalidad y coherencia de su cuerpo teórico, de ahí que la didáctica de las ciencias se suele considerar como una disciplina emergente (Porlán, 1993a) y posible (Aliberas et al., 1989). Sin embargo, no siempre es éste el principal argumento esgrimido para defender una visión de lo científico más próxima a las ciencias experimentales que a las sociales. Las diferencias evidentes entre los objetos de estudio de unas y otras es, a nuestro entender, el núcleo central de la problemática.

Los defensores de que las ciencias experimentales son el prototipo a irmitar suelen basarse en el rigor, Ia objetivi- dad y la universalidad que atribuyen al método científico-experimental. Las posibilidades de manipulación del objeto de estudio hacen albergar la ilusión de que se controla la complejidad de la materia y de los fenómenos con los que se trabaja. Evidentemente, esto no ocurre, ni puede ocurrir, en el caso de seres humanos o de sistemas sociales. La paradoja surge cuando, al analizar el discurso de la ortodoxia cientificista, descubrimos, bajo la superficie de los argumentos anteriores, que las razones para cuestionar el carácter científico de determinadas disciplinas son en realidad razones que dan fe de una concepción simplificadora e ingenua del trabajo científico y, por añadidura, de la naturaleza del conocimiento que se pretende defender. Si lo que caracteriza el trabajo científico es fundamentalmente la mayor o menor capa. cidad de controlar la realidad material, en el sentido de someterla a situaciones artificiales que nos permitan ensayar relaciones causa-efecto de carácter lineal y descubrir leyes generales, no sólo quedarían fuera de la ciencia las disciplinas humanas y sociales, sino también muchas de las mal Ilamadas experimentales. La geología, buena parte de la biología, la física teórica, etc. no producen conocimiento bajo estas premisas. En realidad, lo que la ortodoxia cientificista defiende no es tanto la pureza de un saber genuinamente científico sino la supremacía del conocimiento positivo sobre cualquier otra forma de conocimiento científico (Habermas, 1968b; Chalmers, 1976; Morin, 1982).

La crítica al positivismo es algo comúnmente aceptado hoy en día entre filósofos y epistemólogos; sin embargo, su influencia social sigue siendo determinante. Hasta tal punto esto es así que el estereotipo social dominante sobre la ciencia, el que poseen muchos profesores y estudiantes, coincide con el reduccionismo experimentalista descrito anteriormente y con un conocimiento positivo, pragmático, empírico y metodológicamente eficaz y seguro, alejado de actividades creativas y espe- 
culativas (Schibeci, 1986; Porlán, 1986, 1989). No obstante, no podemos ignorar el problema real de la excesiva ligere za con que determinadas teorías, con un origen claramente filosófico o especulativo (véanse los casos emblemáticos del marxismo y del psicoanálisis), se han venido autodenominando como científicas. Es evidente que la ampliación del campo de lo científico no puede ser a costa de su desnaturalización epistemológica, pero también lo es que la mejor manera de defenderse de las pseudociencias (Bunge, 1985) no es a través de un proceso đe reducción y de simplificación esquemática de los procesos científicos. La apuesta, conveniente y deseable, por ampliar los ámbitos de la realidad susceptibles de ser abordados científicamente no se abrirá camino ni desde el absolutismo positivista, con su empeno por establecer jerarquías epistemológicas rígidas, $\mathrm{ni}$ desde el relativismo radical del todo vale (Feyerabend, 1975), tendente a eliminar cualquier críterio de racionalidad científica.

En este sentido, es conveniente analizar los planteamientos epistemológicos de Toulmin (1972), ya que dicho autor describe, desde una perspectiva integradora, alejada de la comparación simplista y excluyente entre ciencias experimentales y sociales, las características comunes a todas las disciplinas profesionalizadas, sean consideradas científicas o no, en contraste con aquellas actividades humanas no profesionales, que no tienen carácter disciplinar. Para este autor las disciplinas son "empresas racionales en evolución" que implican un proceso de selección y comprensión colectiva de «poblaciones conceptuales». En concreto, dichas características son: a) un conjunto de problemas específicos, conceptuales o prácticos; $b$ ) la existencia de una comunidad profesional crítica; c) un punto de vista general y compartido sobre la disciplina (metas e ideales); d) estrategias y procedimientos aceptados; y e) poblaciones conceptuales en evolución vinculadas a los problemas específicos. Atendiendo a estos criterios, el autor establece tres grupos diferentes de disciplinas: las compactas o maduras (cuando reúnen todos los requisitos); las difusas (cuando sólo reúnen algunos de ellos) y las posibles (cuando pueden llegar a reunirlos). Toulmin distingue también entre disciplinas explicativas, que, como su nombre indica, tienen como meta la descripción y explicación de los problemas específicos que les atañen, y disciplinas prácticas, que pretenden la resolución de problemas prácticos y, en consecuencia, la orientación rigurosa de procesos concretos de transformación de la realidad.

En un trabajo anterior (Porlán 1993b) ya planteábamos la relevancia del enfoque epistemológico de Toulmin para superar las versiones más extremas del absolutismo y del relativismo epistemológico, así como la pertinencia de este punto de vista para dar luz sobre diversos problemas relevantes de la enseñanza-aprendizaje de las ciencias. No nos detendremos ahora en analizar los diferentes aspectos en que se puede manifestar esta influencia enriquecedora y positiva, simplemente utilizaremos los mismos argumentos de entonces para plantear que la diđáctica de las ciencias es una disciplina emergente (cumple algunos de los requisitos expuestos), posible (su evolución indica que puede llegar a cumplirlos todos) y práctica (sus problemas específicos se refieren a la educación científica). En los apartados siguientes profundizaremos más en algunos de estos aspectos.

\section{ANTECEDENTES DE LA DIDÁCTICA DE LAS CIENCIAS}

Aun cuando existen precedentes históricos anteriores (véase el caso, por ejemplo, de la revista Science Education, que se publica desde el año 1917), para diversos autores (Gutiérrez, 1987; Aliberas et al. 1989; Cañal, 1990), el origen de la didáctica de las ciencias como área de conocimiento disciplinar hay que situarlo en los años cincuenta, asociado al desarrollo institucional que en los países anglosajones se da a la investigación y experimentación en el campo de la enseñanza de las ciencias y en el contexto de una serie de medidas político-económicas y educativas que pretenden impulsar el crecimiento científico y tecnológico de estos países (Fig. 1). En concreto, en Estados Unidos se ponen en marcha durante este periodo propuestas curriculares que pretenden transformar la enseñanza tradicional de las ciencias, con fuerte apoyo económico y con la implicación de científicos, psicólogos y pedagogos de prestigio. Recuérdese, por ejemplo, el Physical Science Study Committee (PSSC), el Chemical Bond Approach Project (CBA), el Biological Sciences Curriculum Study (BSCS), el Elementary Science Study (ESS) o el Science Curriculum Improvement Study (SCIS). Todo ello da lugar al denominado movimiento de reforma curricular que se extiende a otras áreas educativas y que acaba constituyéndose en un auténtico proceso de reforma del sistema educativo en su conjunto (Fernández Uría, 1979). En Inglaterra, durante los mismos años, se ponen en marcha ambiciosos proyectos curriculares para la enseñanza de las ciencias (p.e., el caso emblemático del programa de la Nuffield Foundation) al mismo tiempo que se presta una atención especial a la formación permanente del profesorado de ciencias. Surgen instituciones como la Association for Science Education que tienen como objetivo fundamental atender y dinamizar dicha formación.

Todo este amplio movimiento de experimentación en torno a la enseñanza de las ciencias da lugar, en paralelo, a un cierto desarrollo conceptual de la didáctica correspondiente, de la misma manera que ocurre en otros ámbitos de las ciencias de la educación. De una manera resumida, la necesidad de un fuerte desarrollo tecnológico en los países occidentales fuerza la progresiva estructuración cientifico-positivista de las disciplinas educativas, extendiéndose al campo de la investigación educativa los enfoques experimentalistas y cuantitativos (Pérez Gómez, 1983).

En el caso de los proyectos curriculares de ciencia se pretende la superación de los enfoques tradicionales, ya que, se argumenta, transmiten una visión fragmentaria y 
caduca de las disciplinas, desprovista de sus andamiajes metodológicos, seleccionándose, según esto, contenidos científicos actualizados y coherentes con la lógica interna de cada disciplina. Se incorpora, así mismo, una metodología didáctica emuladora de la concepción inductivista del método científico. Se elaboran, por último, taxonomías de objetivos científicos que pretenden desarrollar operativamente las competencias conceptuales, procedimentales y actitudinales de los estudiantes (Moore y Sutman, 1970; Klopfer, 1971 y Bloom, 1972).

Diversos autores (Gutiérrez, 1987, Astolfi y Develay, 1989: Cañal, 1990) señalan el final de los años setenta y comienzo de los ochenta como el momento en que se produce una crisis de la tendencia cientificista y tecnológica de la didáctica de las ciencias. Una vez más, factores sociales y políticos determinan, en parte, la nueva situación. En los países occidentales comienza a ponerse en duda la posibilidad de un desarrollismo ilimitado, los problemas ambientales y la posibilidad de una guerra nuclear alertan a la población sobre la aparente bondad y universalidad del trabajo científico y el ideal de una ciencia objetiva, neutral y verdadera se desvanece ante la evidencia de los intereses económicos, sociales y profesionales implicados (Fig. 1). La crisis del positivismo científico-técnico no sólo se manifiesta en un plano social, también to hace en el campo de la reflexión filosófica y epistemológica y, desde luego, en el interior de las propias disciplinas científicas. En el caso de las ciencias sociales, por ejemplo, se manifiestan críticas epistemológicas basadas en perspectivas fenomenológicas y relativistas, desarrollándose nuevas líneas de investigación que intentan superar los planteamientos de caja negra tan frecuentes hasta ese momento, atendiendo más al significado de los sujetos que a la supuesta objetividad y neutralidad del proceso de investigación. En la misma línea, se cuestiona la creencia ingenua de que los principios de la racionalidad tecnológica (planificación minuciosa y cerrada de los procesos productivos, secuencia única de tareas, control estricto y objetivo de la eficacia de dichos procesos, etc.) son aplicables a los procesos humanos y sociales (funcionamiento de las instituciones, procesos educativos, terapias psicológicas, etc.).

En el ámbito didáctico general, se produce un movimiento de crítica a los enfoques de investigación estadístico-cuantitativos y a los modelos simplificadores que consideran que la enseñanza causa el aprendizaje, modelo proceso-producto (Guba, 1981; Pérez Gómez, 1983; Porlán, 1989; Contreras, 1991). Se resaltan, por contraposición, los enfoques holisticos y situacionales, se proponen metodologías cualitativas y de estudio de casos y se comienzan a valorar los significados construidos por profesores y alumnos como variables mediadoras en el proceso de enseñanza-aprendizaje (Shavelson y Stern, 1981; Shulman, 1981; Yinger y Clark, 1982; San Martín, 1984; Clark, 1985; Pérez Gómez y Gimeno, 1988; Porlán, 1989).

En la didáctica de las ciencias, las aportaciones de la nueva epistemologia de la ciencia influyen de manera determinante (López Rupérez, 1990). Autores como
Kuhn(1962), Toulmin (1972), Feyerabend(1975), Lakatos (1978) y otros ponen en evidencia los errores del positivismo y de otras creencias asociadas (inductivismo, realismo, absolutismo, etc.). En general, estas reflexiones abren un debate importante acerca de la naturaleza de las teorías científicas, de su carácter relativo y evolutivo, y del papel que juegan al condicionar y dirigir, inevitablemente, todo proceso de observación, interpretación e intervención en la realidad. Esta visión más dinámica y contextualizada de la ciencia pone en crisis muchas de las presunciones del movimiento de reforma del currículo científico. Ya no se trata de enseñar una ciencia absoiuta, sino tentativa, condicionada por intereses sociales y sometida a procesos más o menos acelerados y profundos de cambio (Abimbola, 1983; Hodson, 1986).

Simultáneamente, desde la psicología del aprendizaje emergen nuevos problemas que trascienden al campo de la didáctica de las ciencias. La crítica al conductismo abre la posibilidad de un abordaje científico del mundo de los significados personales (Kelly, 1955; Ausubel, 1968; Kelly, 1970; Fransella, 1978; Claxton, 1984; Vega, 1984). Desde la psicología cognitiva se trata de establecer un programa de investigación que, sin caer en la tentación especulativa del psicoanálisis, dé cuenta de las variables internas de los sujetos, no sólo para analizar la estructura de sus sistemas de ideas (Norman, 1982), sino también para describir los contenidos concretos que los conforman.

En general, la crisis de la racionalidad científico-técnica provoca una redefinición teórica y metodológica de ia didáctica de las ciencias, que se orienta hacia una visión más fenomenológica del objeto de estudio, hacia metodologías más abiertas y cualitativas y hacia una concepción más relativa đel conocimiento.

En España, aunque con retraso, tambiến se vive durante la đécada de los ochenta un desarrollo importante de esta disciplina. Se establecen grupos y líneas de investigación más o menos estables, se editan revistas relacionadas con la temática (Cuadernos de Pedagogía, Enseñanzade las Ciencias, Investigación en la Escuela, Alambique, etc.) y se realizan congresos periódicos en lo que va tomando cuerpo una comunidad científica incipiente (Jiménez 1988; Cañal 1990).

Según Astolfi y Develay (1989), entre los años 1980 y 1985 se realiza por parte de la comunidad científica internacional un proceso de integración y reelaboración de tres líneas de investigación presentes en el campo: a) una línea más epistemológica, que trata de hacer una lectura didáctica de la estructura de los contenidos científicos y de los problemas, obstáculos y perspectivas más relevantes de su evolución histórica; $b$ ) una más psicológica, que trata de describir las concepciones que tienen los estudiantes sobre los fenómenos de la realidad, las características de dichas concepciones y los procesos a través de los cuales se producen y evolucionan; y c) una línea pedagógica de carácter más general que se plantea la estructura y la dinámica de la comunicación en el aula de ciencias. Este proceso de reconceptualización va 
acompañado de una nueva definición de los objetivos de la enseñanza de las ciencias. Si en la década de los sesenta y los setenta, al calor del desarrollismo tecnológico, lo importante era enseñar más y mejor ciencia, con la idea de aumentar la capacidad de producción científica y tecnológica de la sociedad, en la década de los ochenta, ante la evidencia de que las tendencias tradicionales y tecnológicas no provocan, en un número importante de casos, aprendizajes significativos en los alumnos (Novak y Gowin, 1984; Giordan y De Vecchi, 1987), empieza a abrirse camino la idea de una enseñanza de las ciencias para todos Ios ciudadanos como medio para democratizar el uso social y político de la ciencia. Las personas, según este nuevo punto de vista, deben comprender para poder decidir, deben desmitificar para poder participar y deben incorporar en su formación básica ciertos aspectos de la cultura científica. Se comienza, por tanto, a cuestionar si el objetivo de la educación científica en las etapas obligatorias es realmente uenseñar las ciencias en sentido estricto» 0 , más bien, contribuir desde las ciencias a la formación general de todos los ciudadanos:

«Este enfoque permite considerar que las preguntas que surgen respecto al cambio de objetivos de una enseñanza de las ciencias (que se ofrece, ahora, a toda la población escolar y que, por lo tanto, se dirige cada vez más a alumnos que nunca serán científicos) son auténticos «problemas científicos». Estos nuevos objetivos y estas nuevas funciones, más vivenciales de la diđáctica de las ciencias, se relacionan con la necesidad de atender las necesidades de formación fundamentales de los ciudadanos y de dar un enfoque más amplio a la enseñanza de las ciencias, que incluya estos nuevos aspectos: los valores relacionados con el vínculo entre ciencia y sociedad; la tecnología; el conocimiento científico en relación a la calidad de vida de los alumnos; la significación personal que todo proceso formativo debe tener para el alumno[...]» (Aliberas et al., 1989, p. 281).

«Cuando damos formación científica, ¿nos limitamos simplemente a crear un mito? Responderé lo que me dijeron los alumnos de primero: La ciencia es seria porque no entiendo nunca nada, aparte de fórmulas, pero me la creo[...] Las consecuencias de este engaño son graves. Favorece el desarrollo de la irracionalidad y permite el secuestro del saber $y$, por ende, del poder» (Giordan, 1978, p. 21).

De todo lo anterior podemos concluir que durante la década de los ochenta la didáctica de las ciencias sufrió un importante proceso de clarificación. Durante este periodo de tiempo pasó de ser un conjunto de prescripciones curriculares que pretendían trasladar a la escuela la lógica de las disciplinas científicas y la versión positivista del método científico, sin tener en cuenta el conjunto de variables mediadoras que intervienen en los sistemas de enseñanza-aprendizaje (concepciones de alumnos y profesores, contexto escolar y extraescolar, etc.), a ser una disciplina posible, en el sentido de disponer, aunque de manera rudimentaria, de algunos de los requisitos imprescindibles para ser considerada como tal (Toulmin, 1972; Bunge, 1976, Fig. 1).

\section{PROBLEMAS ACTUALES Y DESARROLLO FUTURO}

Después de la breve descripción histórica realizada en el apartado anterior, conviene ahora formalizar las características fundamentales que definen la didáctica de las ciencias en el momento presente, así como los problemas y líneas de investigación más fructifferos que pueden impulsar su desarrollo futuro.

Consideramos la didáctica de las ciencias como una disciplina práctica emergente que se incluye en el campo más amplio de las ciencias de la educación. Aun cuando su origen está más vinculado a las ciencias experimentales, actualmente, después de un amplio proceso de reflexión y reelaboración epistemológica, psicológica y didáctica, está plenamente integrada en el conjunto de disciplinas que se interesan por los problemas educativos. En gran medida, este proceso de redefinición, que ha estado inmerso en un cambio mucho más amplio y profundo đe carácter social, económico, político y filosófico, ha marcado la evolución de sus cuarenta y cinco años de existencia más o menos formal.

El objeto de estudio de la didáctica de las ciencias son los sistemas de enseñanza-aprendizaje, en tanto que en ellos se aborden fenómenos materiales y naturales. No obstante, tal como decíamos al principio, el carácter práctico de esta disciplina hace que su finalidad no se limite a la descripción y explicación de dichos sistemas, sino que abarque también aspectos relacionados con la valoración y transformación de los mismos atendiendo a criterios de calidad y de coherencia con los objetivos generales de la educación (Astolfi, 1994). Teniendo esto en cuenta, la finalidad de la didáctica de las ciencias presenta dos dimensiones complementarias: describir y analizar los problemas más significativos de la enseñanzaaprendizaje de las ciencias y elaborar y experimentar modelos que, a la luz de los problemas detectados, ofrezcan alternativas prácticas fundamentadas y coherentes. Para conseguir desarrollar esta doble finalidad, ha de plantearse un conjunto de probiemas prioritarios y líneas de investigación. En este sentido, existen en la literatura un número significativo de autores que han realizado aportaciones valiosas sobre este asunto, algunas de las cuales analizaremos a continuación.

Es ya clásica la relación que estableció Linn (1987) sobre líneas prioritarias de investigación, que ha servido de obligado punto de referencia para reelaboraciones posteriores, especialmente en el mundo anglosajón. En concreto, este autor propone, entre otras de menor entidad, las siguientes problemáticas básicas para el desarrollo de la didáctica de las ciencias: a) identificar nuevas metas en la enseñanza de las ciencias; $b$ ) desarrollar y refinar nuestro conocimiento sobre la enseñanza y la instrucción actuales; $c$ ) desarroliar y poner a prueba currículos experimentales alternativos; $d$ ) evaluar Ia efectividad de estas innovaciones; $e$ ) desarrollar y evaluar nuevas metodologías para estimar los aprendizajes de los estudiantes; $y f$ ) diseñar y evaluar nuevos modelos de formación del profesorado para la enseñanza de las ciencias. 
Figura 1

Evolución de la didáctica de las ciencias.

\section{ETAPA PREDISCIPLINAR}

- VISIÓN DEFORMADA DE LA CIENCIA

- AUSENCIA DE INVESTIGACIÓN DIDÁCTICA

- NO RECONOCIMIENTO DISCIPLINAR

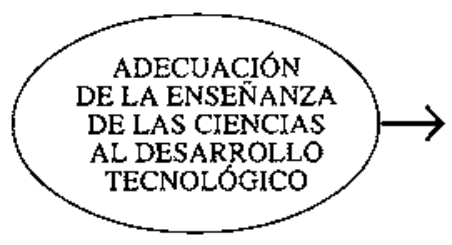

\section{ETAPA TECNOLOGICA}

- VISION POSITIVISTA DE LA CIENCIA

- VISIÓN SIMPLIFICADA DE LOS PROCESOS DE ENSENAANZA-APRENDIZAJE DE LAS CIENCIAS

- LOGICA CIENTÍFICA COMO PRINCIPIO DIDÁCTICO

- didÁctica de las cIENCIAs VINCUlada a LA REFORMA CURRICULAR

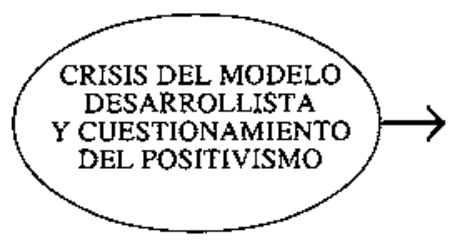

ETAPA ACTUAL

- VISIÓN MÁS RELATIVISTA DE LA CIENCIA

- VISIÓN MÁS COMPLEJA DE LOS PROCESOS DE ENSENAANZA-APRENDIZAIE DE LAS CIENCLAS

- ESTUDIO DELASCONCEPCIONES DELOS ALUMNOS

- CRÍTICA Al MODELO TRADICIONAL Y AL TECNOLÓGICO

- APERTURA INTERDISCIPLINAR

- ANÁlISIS DIDÁCTICO DE LOS CONTENIDOS

- estudio inICIAL DE las CONCEPCIONES DE LOS PROFESORES

- MODELO ALTERNATIVO CONSTRUCTIVISTA

- DIDÁCTICA DE las CIENCIAS COMO DISCIPLINA PRACTICA
Resulta de igual interés, aun cuando no recoja la diversidad de líneas que de hecho existen, la síntesis que hacen Astolfi y Develay (1989) refiriéndose a las aportaciones de otros autores como Host (1978) y Tiberghien (1985), síntesis que es bastante representativa de las preocupaciones existentes en el ámbito francófono. Estos autores establecen cuatro áreas fundamentales de investigación: a) investigaciones en torno a la construcción y evaluación de un modelo didáctico alternativo; b) investigaciones sobre los contenidos disciplinares, derivando en investigaciones curriculares; $c$ investigaciones sobre los métodos de enseñanza de las ciencias, articulando las dimensiones epistemológicas, psicológicas y de interacción social (estudios de representaciones de los alumnos, aprendizaje por resolucion de problemas, etc.); y $d$ ) investigaciones sobre la organización del aula en relación con propuestas innovadoras concretas.

Como se puede observar, ambas propuestas dan por supuesto la necesidad de un cambio profundo en la enseñanza actual de las ciencias, cambio que, como comentábamos al final del apartado anterior, se viene gestando desde comienzo de los ochenta. Ni los enfoque tradicionales y enciclopédicos, aun hoy predominantes a pesar de los hallazgos de la investigación didáctica, ni Ios enfoques cientificistas que surgen en la década de los setenta, dan respuestas de calidad al evidente fracaso de la enseñanza de las ciencias desde un enfoque internacional. Los autores mencionados ponen el énfasis en la elaboración y experimentación de modelos y currículos alternativos que presten atención especial a las variables mediadoras relacionadas con los alumnos (concepciones alternativas, obstáculos de aprendizaje, dimensión social del aprendizaje, contexto educativo, etc.). Linn introduce además otro aspecto que se ha convertido en la línea de investigación más pujante de los últimos cinco años: los estudios relacionados con el profesor de ciencias.

En nuestro país encontramos también aportaciones valiosas sobre los problemas y enfoques prioritarios para la investigación. Cañal (1990), por ejemplo, plantea tres cuestiones básicas para incidir en la mejora de la enseñanza de las ciencias, poniendo un acento especial en eI desarrollo profesional de los profesores de ciencias: a) el desarrollo progresivo de un cuerpo teórico coherente y funcional; $b$ ) el desarrollo de propuestas y materiales curriculares para los diversos niveles, disciplinas, tópicos, centros de interés, etc., como recurso necesario para la concreción por los profesores de diseños curriculares más contextualizados; y c) la evaluación de estas propuestas curriculares, perfeccionándolas, y atendiendo especialmente a la mejora de la labor profesional del enseñante implicado en las mismas.

Furió y Gil (1989), en un artículo donde reflexionan sobre la relación entre la didáctica de las ciencias y la formación del profesorado, aportan una agenda muy detallada del estado de la cuestión: a) profundizar en los estudios sobre la construcción y el aprendizaje de conceptos, especialmente el papel de las concepciones previas de los alumnos en el proceso de aprendizaje; b) iniciar estudios sobre las preconcepciones científicas 
y didácticas del profesorado y su papel en la formación inicial y permanente; c) promover la familiarización de los alumnos con las características del trabajo científico; d) considerar la resolución de problemas como punto de partida para la construcción de conocimientos científicos en la escuela; $e$ ) investigar la actitudes hacia la ciencia y su aprendizaje, incluyendo el estudio de las diferencias de actitud entre alumnos y alumnas, y sus causas; $f$ ) potenciar las relaciones entre enseñanza de las ciencias y medio, incluyendo los planteamientos ciencia-técnica-sociedad; $g$ ) analizar el clima del aula de ciencias, las formas de trabajo de los alumnos y los roles del profesor; $h$ ) considerar la evaluación como instrumento esencial de seguimiento del proceso de aprendizaje y de la mejora de la enseñanza; $i$ ) establecer criterios para el establecimiento de un currículo de ciencias alternativo; j) analizar los diferentes modelos vigentes de enseñanza-aprendizaje de las ciencias y su fundamentación.

En esta propuesta se puede observar claramente como, en coherencia con lo expuesto acerca de la doble finalidad de la didáctica de las ciencias, aparecen mezcladas líneas de investigación explicativas con propuestas innovadoras que pretenden incidir en la mejora de la enseñanza de las ciencias y que se plantean a efectos de su experimentación y seguimiento en situaciones prácticas. Estando de acuerdo con el carácter tcórico-práctico de esta disciplina, consideramos necesario, no obstante, plantear las propuestas innovadoras en el marco de proyectos curriculares integradores y alternativos que eviten, en lo posible, la excesiva fragmentación y compartimentalización de las líneas prácticas de investigación. Así por ejemplo, el apartado i) de esta propuesta abarca, o debería abarcar, en sí mismo, buena parte de los otros apartados.

Estos mismos autores, en posteriores trabajos, presentan análisis mucho más elaborados y actualizados de los problemas de investigación. Por ejemplo, Furió (1994) plantea que durante los últimos veinte años la investigación en didácticas de las ciencias se ha focalizado en el aprendizaje de los alumnos y en el estudio de sus concepciones alternativas en diversos ámbitos de las ciencias. Los resultados de estas investigaciones ponen en evidencia la enorme disparidad existente entrc los significados reales de los alumnos y los supuestos aprendizajes que deberían haber adquirido con la enseñanza recibida. Esto demuestra la ineficacia del modclo tradicional de enseñanza de las ciencias y la poca influencia que todavía tienen los modelos alternativos (Fig. 1). Según Furió, el hecho de que los profesores reinterpreten las orientaciones curriculares, incluso aquéllas más novedosas, desde puntos de vista fuertemente influidos por el peso de la tradición, y de que, por tanto, las innovaciones didácticas tengan dificultad para Ilegar a las aulas, está provocando un desplazamiento de las prioridades de investigación en la didáctica de las ciencias hacia el estudio de la enseñanza y, en particular, hacia el de su principal protagonista: el profesorado. En este sentido el autor indica que «se está pasando de investigar lo que piensa y hace el alumno en clase hacia lo que piensa y hace el profesor, tratando de analizar sus actividades y así poder descifrar las claves de su desarrollo profesionals (p. 188).

En el mismo sentido, Gil (1994) plantea que el estudio de las concepciones de los alumnos «ha constituido la línca prioritaria de investigación en didáctica de las ciencias durante la década de los ochenta", como lo demuestra la revisión de Pfundt y Duit (1993), que incluye cerca de 3.000 trabajos sobre la temática. Según este autor, con cl que coincidimos, las aportaciones más relevantes de esta corriente de investigación han sido las siguientes: $a$ ) ha cuestionado la validez de los modelos de enseñanza de las ciencias por transmisión de conocimientos elaborados, poniendo en crisis la visión simplista según Ia cual, para enseñar, basta con conocer la materia y adquirir cierta experiencia; b) ha integrado diferentes perspectivas teóricas, especialmente los enfoques lingüísticos, psicológicos y epistemológicos; c) ha hecho posible la emergencia de un consenso teórico en torno al constructivismo, como fundamento de un modelo alternativo para la enseñanza-aprendizaje de las ciencias; d) ha puesto en evidencia la importancia de los estudios sobre la génesis histórica de los conocimientos científicos y la relevancia de la fílosofia de las ciencias para la conseñanza de dichas disciplinas; $e$ ) ha favorecido el surgimiento de nuevos modelos de enseñanza de las ciencias que pretenden promover el aprendizaje de los alumnos a través de la investigación dirigida de problemas de interés; $f$ ) por último, ha dirigido la atención hacia las concepciones epistemológicas y didácticas de los profesores en formación y en ejercicio, de manera que, por analogía, se ha llegado a comprender que dichas concepciones, especialmente las adquiridas por impregnación ambiental cuando fueron alumnos, pueden constituir un importante obstáculo para la evolución de la enseñanza de las ciencias.

Por lo tanto, nos encontramos ante una línea de investigación consolidada, centrada en la descripción y el análisis del pensamiento de los alumnos (Martinand, 1996), que ha organizado una parte importante de la actividad de reflexión e investigación de la disciplina durante los últimos quince años, y una línea de investigación emergente que, ante las dificultades encontradas para conectar los resultados de los estudios anteriores con la actividad docente, ha fijado su atención en el conocimiento y en la práctica de los profesores, así como en los modelos y estrategias que pueden favorecer su desarrollo profesional (Develay, 1996).

Por nuestra parte, hemos venido planteando, en diferentes trabajos (Porlán, 1993a; Porlán, 1993c; Porlán y Martín, 1994), un conjunto de reflexiones sobre la evolución de esta disciplina y sobre los problemas y estrategias que han impulsado y pueden impulsar su desarrollo futuro. Desde nuestro punto de vista, la diđáctica de las ciencias ha experimentado un progreso notable en los últimos veinte años en la medida que ha elaborado y sistematizado una crítica rigurosa a los enfoques dominantes en la enseñanza de las ciencias. Es decir, su emergencia como disciplina práctica, y la consiguiente superación de periodos anteriores donde predominaban los aspectos exclusivamente normativos y donde no 
existía una investigación disciplinar propiamente đicha, ha ido produciéndose en relación con los procesos de reflexión y teorización crítica sobre el modelo mayoritario de enseñanza-aprendizaje de las ciencias.

En un trabajo anterior (Porlán y Martín, 1994) hemos destacado las siguientes deficiencias de la enseñanza habitual de las ciencias: a) convierte directamente los contenidos de las disciplinas científicas en contenidos curriculares, sin que medie ningún tipo de reflexión acerca de la naturaleza epistemológica del conocimiento que se pretende promover en la escuela; $b$ ) implica y favorece una visión fragmentaria, acumulativa y absoluta de la ciencia y de los contenidos escolares; $c$ ) ignora los aspectos éticos, actitudinales y procedimentales de la educación, sin tomar en consideración las aportaciones que la ciencia puede hacer en estos campos; $d$ ) considera a los alumnos receptores pasivos de información, como si no tuvieran experiencias y significados espontáneos sobre Ios fenómenos naturales; $\boldsymbol{e}$ ) separa los contenidos de la metodología didáctica y de la evaluación, como si entre los procesos de producción y regulación de significados y los significados mismos no hubiera relaciones de interdependencia; $f$ ) ignora la dimensión social y colectiva del aprendizaje; $g$ ) concibe la evaluación como una actividad selectiva y sancionadora, que suele centrarse en la medición de aprendizajes memorizados mecánicamente por los alumnos.

El énfasis puesto por la didáctica de las ciencias en la crítica rigurosa a los enfoques transmisivos ha estado estrechamente vinculado a los avances en los estudios sobre concepciones de los alumnos, lo que probablemente explique por qué han sido predominantes durante tantos años. Estos estudios, por ejemplo, han puesto en cuestión la idea de que los alumnos son receptores pasivos de la información al demostrar que tienen concepciones espontáneas sobre el mundo y que dichas concepciones suponen, con frecuencia, una alternativa diferente a la científica para explicar los fenómenos de la realidad. Al mismo tiempo, la persistencia de muchas de estas concepciones a lo largo del tiempo, con independencia del éxito o el fracaso académico de los alumnos, confirma la poca eficacia de la enseñanza recibida y el hecho de que la evaluación no informe realmente sobre lo que el alumno verdaderamente sabe, sino sobre Io que aparenta que sabe. La existencia de estas concepciones, en fin, refuerza la idea de que es necesario teorizar sobre la naturaleza epistemológica del conocimiento escolar, pues ya no es posible considerar la ciencia como única fuente de contenidos, dado que los alumnos contienen también una visión del mundo que el currículo debe considerar (Fig. 1).

La caracterización y el análisis de las ideas de los alumnos sobre multitud de tópicos curriculares ha producido un doble efecto en la comunidad investigadora. Por un lado se han ampliado las perspectivas teóricas hacia nuevos dominios epistemológicos y psicológicos, produciéndose lo que Astolfi y Develay (1989) denominan una fuerte apertura interdisciplinar, con lo cual se ha tratado de elaborar una alternativa para la enseñanza de las ciencias que tuviera en cuenta las características de las concepciones de los alumnos. En este sentido, la teoría constructivista ha jugado un papel esencial como fundamento básico de los nuevos enfoques. Por otro, se han puesto en marcha experiencias de aula que han intentado movilizar y hacer evolucionar las ideas de los alumnos, provocando su cuestionamiento y detectando los obstáculos que impiden su evolución hacia formulaciones más adecuadas y complejas. Indudablemente estos dos procesos, el de reconceptualización teórica y el de experimentación práctica, no han ocurrido de manera aislada, sino que se han visto sometidos a una progresiva interdependencia mutua. Sin embargo, los intentos por generalizar un modelo constructivista de enseñanza de las ciencias han encontrado importantes obstáculos. El hecho de que la profesión docente esté radicalmente fragmentada en dos sectores con actividades y exigencias profesionales diferentes (los profesores universitarios, con tiempo para la investigación y la reflexión teórica, y el resto de los profesores, dedicados exclusivamente a la docencia) y la creencia ingenua de que los hallazgos procedentes de la investigación didáctica se pueđen transmitir linealmente a la práctica escolar son algunas de las causas más importantes que explican las dificultades para hacer avanzar la enseñanza de las ciencias en la dirección esperada.

Paradójicamente, los mismos enfoques que enfatizan la inutilidad de pretender enseñar algo a los alumnos ignorando sus concepciones espontáneas han sido transmitidos a los profesores ignorando sus concepciones docentes y sus limitaciones laborales y contextuales. De ahí que, en los último cinco años, haya habido un incremento significativo de las investigaciones y reflexiones centradas en el profesor. De nuevo los estudios sobre las concepciones y obstáculos, en este caso de los profesores, centran la atención inicial de esta línea emergente de investigación. En menor medida comienzan a aparecer también propuestas de formación alternativas basadas en un enfoque constructivista del aprendizaje profesional. En nuestro caso estamos doblemente comprometidos e implicados en esta perspectiva: como formadores de profesores y como investigadores.

En resumen, podemos afirmar que el desarrollo futuro de la didáctica de las ciencias ha de organizarse, a nuestro entender, en torno a cuatro problemas interrelacionadas (frente a la tendencia de diferentes autores a presentar listas desestructuradas de líneas de investigación, hemos preferido organizar los problemas en cuatro categorías generales que resalten las cuestiones más esenciales) (Fig. 2):

a) Profundizar en los fines y fundamentos de un modelo alternativo de enseñanza-aprendizaje de las ciencias. Desde nuestro punto de vista, el constructivismo no resueive todos los problemas de la enseñanza por transmisión de contenidos elaborados. La confluencia en la escuela de epistemologías y culturas diferentes (la cient́fica, la cotidiana, la escolar, la profesional, etc.) y la pretensión de la educación obligatoria de favorecer el desarrollo armónico de los alumnos, y no su especialización disciplinar, plantean un conjunto de dilemas que desbordan los límites estrictos deI constructivismo. En 
Figura 2

Desarrollo futuro de la didáctica de tas ciencias.

\section{PROBLEMA CENTRAL}

- DIFICULTADES PARA EXTENDER EL MODELO CONSTRUCTIVISTA DE ENSEÑANZA DE LAS CIENCIAS

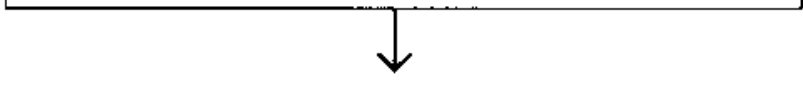

LINEAS DE INVESTIGACION

- PROFUNDIZAR EN LOS FINES Y FUNDAMENTOS - desarRollar UNA TEORfa dEL CONOCIMIENTOESCOLAR

- DESARROLlaR UNA TEORIA DEL CONOCIMIENTOPROFESFNAL

- EXPERIMENTAR PROPUESTAS DE FORMACION PARA PROMOVER, A SU VEZ, LA EXPERIMENTACIÓN DE LOS PROFESORES este sentido, la teoría sistémica, la teoría de la complejidad, la teoría evolucionista y la teoría crítica son, entre otras, aportaciones relevantes.

b) Desarrollar una nueva teoría del conocimiento escolar y de las estrategias que favorecen su construcción. Esta teoría ha de integrar los resultados de los estudios sobre las concepciones y obstáculos de los alumnos, sobre el análisis diđáctico de la historia y epistemología de las ciencias y sobre las implicaciones educativas de las relaciones entre ciencia, técnica y problemática socioambiental. Así mismo, ha de establecer hipótesis coherentes sobre la organización y evolución de dicho conocimiento, así como sobre las pautas metodológicas y evaluativas que lo facilitan.

c) Desarrollar una nueva teoría del conocimiento profesional y de las estrategias que favorecen su construcción. De la misma manera que en el punto anterior, dicha teoría ha de integrar los resultados de Ios estudios sobre concepciones y obstáculos de los profesores, sobre el análisis didáctico de las fuentes disciplinares y sobre los problemas prácticos de los profesores. Así mismo, ha de establecer hipótesis coherentes sobre la posible organización y evolución de dicho conocimiento, así como sobre las estrategias metodológicas y evaluativas que lo facilitan.

d) Diseñar y experimentar propuestas de formación del profesorado que, tomando como referencia los avances en los puntos anteriores, tengan como orientación fundamental promover y apoyar, a su vez, la experimentación de hipótesis curriculares superadoras de los problemas que plantea el modelo tradicional de enseñanza-aprendizaje de las ciencias. Los resultados de este proceso de experimentaciones encadenadas deben traducirse progresivamente en materiales formativos y curriculares que favorezcan el contraste práctico profesional de formadores y profesores, y la progresiva ampliación del campo de influencia de los modelos alternativos.

Por último, el desarrollo histórico de una disciplina no se basa sólo en la adecuada selección y formulación de problemas. En paraleto con esta definición de los campos prioritarios de investigación, es necesario impulsar estrategias profesionales $\mathrm{e}$ institucionales que mejoren las condiciones materiales, contextuales y personales de investigación (Fig. 2). En este sentido, la consolidación futura de la didáctica de las ciencias como disciplina requiere de avances en los siguientes aspectos:

a) Incrementar el grado de apertura interdisciplinar (Gutiérrez, 1987; Astolfi y Develay, 1989). Frente a la situación actual, en la que predominan dentro de la comunidad científica personas procedentes de licenciaturas en ciencias (biólogos, físicos, químicos, etc.), es necesario crear mayores y mejores mecanismos de comunicación con otros campos disciplinares epistemológicamente próximos (Martinand, 1988), al mismo tiempo que se incorporan a los equipos de investigación especialistas en las diferentes ciencias de la educación que se interesen por los problemas específicos de esta disciplina.

b) Modificar la estructura profesional de la disciplina. La comunidad científica que trabaja en este campo tiene características especiales. La mayor parte de sus miembros son profesores de ciencias de secundaria y profesores universitarios de didáctica de las ciencias y, en menor medida, maestros de primaria. Esta composición internivelar, tan alejada de las estructuras académicas clásicas, encierra grandes ventajas, pero también enormes inconvenientes. Entre las ventajas cabe destacar el hecho de que existe ya un sector minoritario del profesorado no universitario que está haciendo realidad el modelo, tantas veces reivindicado, de profesor. investigador. Entre los inconvenientes hay que destacar la falta de tradición en el trabajo científico que presenta esta incipiente comunidad investigadora. La falta de estructuras estables que den soporte y continuidad a las diferentes líneas de investigación se convierte en un obstáculo, a veces insalvable, para el desarrollo de la disciplina. En este sentido, los departamentos universi- 
tarios de didáctica de las ciencias tienen una responsabi* lidad evidente en la mejora de esta situación. La universidad, con su secular desconexión de los problemas sociales y, en nuestro caso, de Ios problemas educativos, no es ningún ejemplo de compromiso con la práctica, tan necesario para un desarrollo no academicista de las disciplinas didácticas. Sin embargo, ciertas tradiciones académicas han preservado, y preservan, aspectos imprescindibles para un trabajo riguroso que supere los límites de la innovación educativa. Al mismo tiempo, Ia estructura universitaria ampara, por principio, la actividad investigadora. Todo ello nos hace plantear la conve niencia estratégica de organizar en torno a los departamentos de didáctica de las ciencias la investigación de equipos de profesores de primaria, de secundaria y de universidad interesados en estas temáticas.

Al mismo tiempo que se avanza en esta dirección, es necesaria una línea de actuación que vaya haciendo posible, de forma gradual, un cambio en el modelo de profesión docente y en la estructura laboral en que dicha profesión se concreta. Medidas realistas que permitan y favorezcan la adscripción temporal de profesores no universitarios al trabajo de investigación en los departamentos didacticos, que disminuyan, así mismo, la carga lectiva de aquellos profesores de primaria y secundaria comprometidos formalmente en proyectos de investigación y que incentiven el currículo investigador de estos profesores son algunos ejemplos de lo que decimos.

c) Mejorar significativamente la organización de la información empírica disponible. Es éste quizás un empeno difícil đe conseguir, en parte porque es subsidiario de los anteriores, en parte porque es un problema de tiempo, experiencia y madurez disciplinar. Lo que queremos resaltar es la urgencia de acometer trabajos de revisión, sistematización y categorización de la información valiosa que se ha producido en los quince últimos años. En este sentido, por ejemplo, es paradigmático el exhaustivo trabajo de Carmichael, Watts, Driver y otros (1990),

\section{REFERENCIAS BIBLIOGRÁFICAS}

ABDMBOLA, I.O. (1983). The Relevance of the «New» Philosophy of Science for the Science Curriculum. School Science and Mathematics, 83(3), pp. 181-193.

ALIBERAS et al. (1989). La didáctica de las ciencias: una empreșa racional. Enseñanza de las Ciencias, 7(3), pp. 277.284

ASTOLFI, J.P. (1994). Didáctica plural de las ciencias. Análisis contrastado de algunas publicaciones de investigación. Investigación en la Escuela, 24, pp. 7-23. en el que se ofrece una clasificación comentada de numerosos estudios realizados, en el mundo anglosajón, sobre las concepciones de los alumnos relativas a diversos tópicos científicos. Es en esta línea en la que proponemos continuar.

d) Iniciar un proceso de convergencia de las técnicas e instrumentos de investigación. Si bien en numerosos trabajos de los últimos años se viene insistiendo en la necesidad de superar la dicotomía entre técnicas cuantitativas y cualitativas đe investigación (Cook y Reichardt, 1982), esto no se viene traduciendo en un esfuerzo paralelo por unificar y consolidar los instrumentos y técnicas de investigación. El hábito de replicar instrumentos elaborados por otros investigadores, o de aplicar, en contextos diferentes, técnicas ya diseñadas, no es aún excesivamente habitual, en nuestro ámbito. Inevitablemente las técnicas e instrumentos cualitativos (observaciones, diarios, entrevistas, cuestionarios abiertos, etc.) requieren de grandes dosis de adecuación al contexto. Pero todos ellos suelen elaborarse con ciertos protocolos semiestructurados y en relación con determinados sistemas de categorías que permiten establecer una conexión rigurosa y flexible entre los problemas, las hipótesis y los fenómenos de la realidad. Pues bien, es el contraste en este nivel el que consideramos necesario para mejorar el control intersubjetivo de los resultados empíricos. En síntesis, si bien existe entre la comunidad científica un nivel de convergencia importante respecto al cuerpo térico y a los enfoques metodológicos integradores, esto no se traduce en una tendencia a compartir técnicas e instrumentos de investigación, quizás porque esto sea, necesariamente, un paso posterior.

\section{NOTA}

Esta publicación es resultado parcial del proyecto PB94-1449 financiado por la CICYT.
ASTOLFI, J.P. y DEVELAY, M. (1989). La didactique des sciences. París: Presses Universitaires de France.

AUSUBEL, D.P. (1968). Educational psychology: a cognitive view. New York: Holt, Rinehart and Winston. (Trad. cast. Psicología educativa: un punto de vista cognoscitivo. México: Trillas, 1976).

BLOOM, 8.S.(1972). Taxonomía de losobjetivos delaeducación: la clasificación de las metas educacionales. Buenos Aires: El Ateneo. (Edición original: Taxonomy of educational 
objetives: the classification of educational goals. Nueva York: McKay Co, 1956).

BUNGE, M. (1976). La investigación cientifica. Barcelona: Ariel.

BUNGE, M. (1985). Seudociencia e ideología. Madrid: Alianza.

CAÑAL, P. (1990). La enseñanza en el campo conceptual de la nutrición de las plantas verdes: Un estudio didáctico en la educación básica. Tesis doctoral idédita. Universidad de Sevilla.

CARMICHAEL, P., WATTS, D.M., DRIVER, R. et al. (1990). Research on students' conceptions in science: a bibliography. Universidad de Leeds.

CHALMERS, A.F. (1976). What is this Thing Called Science? Queensland: University of Queensland Press. (Trad. cast ¿Qué es esa cosa llamada ciencia? Madrid: Siglo XXI).

CLARK, M. (1985). Ten Years of Conceptual Development in Research on Teacher Thinking. Conference of "International Study Association on Teachers Thinking». Tilburg.

CLAXTON, G. (1984). Live and Learn. An Introduction to the Psychology of Growth and Change in Everyday Life. Londres: Harper and Row Publishers. (Trad. cast. Vivir y Aprender. Psicología del desarrollo y del cambio en la vida cotidiana. Madrid: Alianza, 1987).

CONTRERAS, J. (1991), Enseñanza, currículo y profesorado. Introducción crítica a la didáctica. Madrid: Akal.

COOK, T.D. y REICHARDT, C.S. (1982). Qualitative and Quantitative Methods in Evaluation Research. Sage Publications Inc. (Trad. cast. Métodos cualitativos y chantitativos en investigación educativa. Madrid: Morata, 1986).

DEVELAY, M. (1996). Didactique et perspectives de formation. Educations, enero, febrero, pp. 49-52.

FERNÁNDEZ URIÁ, E. (1979): Estructura y didactica de las ciencias. Madrid: MEC.

FEYERABEND, P. (1975). Against Method. Londres: NLB. (Tad. cast. Tratado contra el método. Madrid: Tecnos, 1981).

FRANSELLA, F. (1978). Personal Construct Psychology 1977. Londres: Academic Press.

FURIÓ, C. (1994). Tendencias actuales en la formación del profesorado de ciencias. Enseñanza de las Ciencias, 12(2), pp. 188-199.

FURIÓ, C. y GIL, D. (1989). La didáctica de las ciencias en la formación inicial del profesorado: Una orientación y un programa teóricamente fundamentados. Enseñanza de las Ciencias, 7(3), pp. 257-265.

GIL, D. (1994). Diez años de investigación en didáctica de las ciencias: realizaciones y perspectivas. Enseñanza de las Ciencias 12(2), pp. 154-164.

GIORDAN, A. (1978). Une Pédagogie pour les Sciences Epérimentales. París: Centurion. (Trad. cast. La enseñanza de las ciencias. Madrid: Siglo XXI, 1982)

GIORDAN, A. y DE VECCHI, G. (1987). Les origines du savoir. Des conceptions des apprenants aux concepts scientifiques. Neuchâtel: Delachaux et Niestlé SA. (Trad. cast. Los origenes del saber. De las concepciones de los alumnos a los conceptos científicos. Sevilla: Díada Editora, 1988).
GUBA, E.G. (1981). Criteria for assessing the truthworthiness of naturalistic inquiries. Anuary, 29 (2), 75-91. (Trad. cast. Criterios de credibilidad en la investigación naturalista, en Gimeno, J. y Pérez, A. La enseñanza: su teoria y su práctica. Madrid: Akal, 1983).

GUTIÉRREZ, R. (1987). La investigación en didáctica de las ciencias. Elementos para stı comprensión. Bordón, 268, pp. 339-362.

HABERMAS,J, (1968). Tecnikund Wissenschaftals "Ideologie». Frankfurt am Main: Suhrkamp Verlag. (Trad. cast. Ciencia y técnica como «ideologia». Madrid: Tecnos, 1986).

HODSON, D. (1986). Philosophy of Science and Science Education. Journal of Philosophy of Education, 20(2), pp. 215-225. (Trad. cast. Filosofía de la ciencia y educación científica, en Porlán, R., García, J.E. y Canal, P. (Comp.). Constructivismo y enseñanza de las ciencias. Sevilla: Díada Editora, 1988).

HOST, V. (1978). Place des procedures d'apprentissages «spontanécs» dans la formation scientifique. Revue de Pédagogie, 45, pp. 103-110. (Trad. cast. El lugar de los procedimientos de aprendizaje «espontáneo» en la formación. científica. Infancia y Aprendizaje, 19-20, pp. 3-20, 1982).

JIMÉNEZ, M.P. (1988). Enseñanza de las ciencias. Cuadernos de Pedagogía, 155, pp. 8-10.

KELLY, G.A. (1955). The Psychology of personal constructs, $I$ and 2. Nueva York: W.W. Norton and Co.

KELLY, G.A. (1970). A brief introduction to personal construct theory, en Bannister, D. (ed.). Perspectives in personal construct theory. Londres: Academic Press.

KUHN, T.S. (1962). The Structure of Scientific Knowledge. University of Chicago Press. (Trad. cast. La estructura de las revoluciones cientificas. México: Fondo de Cultura Económica, 1975).

KLOPFER, L.E. (1971). Evaluation of learning in Science, en Bloom, B.S., Hastings, J. T. y Madaus, G.F. (eds.). Handbook on formative and summative evaluation of student learning. Nueva York: McGraw Hill, pp. 559-641.

LAKATOS, I. (1978). The methodology of scientific research programmes. Philosophical papers, vol. 1. Cambridge: Cambridge University Press. (Trad. cast. La metodología de los programas de investigación científica. Madrid: Alianza Universidad, 1983).

LINN, M.C. (1987). Establishing a research base for science education: challenges, trends and recommendations. Journal of research in science teaching, 24 (3), pp. 191-216.

LÓPEZ RUPÉREZ, E. (1990). Epistemología y didáctica de las ciencias. Un análisis de segundo orden. Enseñanza de las Ciencias, 8(1), pp. 65-74

MARTINAND, J,L, (1988). Cuestiones actuales de la didáctica de las ciencias físicas en Francia: observaciones comparativas. Enseñanza de las Ciencias, 6(1), pp. 47-53.

MARTINAND, J.L. (1996). D'où est venue la Didactique? Educations, enero, febrero, pp. 22-25.

MOORE, R.W. y SUTMAN, F.X. (1970). The development, field test and validation of an inventory of scientific attitudes. Journal of research in science teaching, Vol. 7, pp. 85-94.

MORIN, E. (1982). Science avec Conscience. París: Librairie Arthème Fayard. (Trad. cast. Ciencia con consciencia. Barcelona: Anthropos, 1984). 
NORMAN, D.A. (1982). Learning and Memory. Nueva York: W.H. Freeman and Company. (Trad. cast. El aprendizaje y la memoria. Madrid: Alianza, 1985)

NOVAK, J.D. y GOWIN, B.D. (1984). Learning how to learn. Cambridge, Ma.: Cambridge University Press. (Trad. cast. Aprendiendo a aprender. Barcelona: Martínez Roca, 1988).

PÉREZ GOMEZ, A. (1983). Paradigmas contemporáneos de investigación didáctica, en Gimeno, J. y Pérez, A. La enseñanza: su teoría y su práctica. Madrid: Akal, 1983.

PÉREZ GOMEZ, A. y GIMENO, J. (1988). Pensamiento y acción en el profesor: de los estudios sobre la planificación al pensamiento práctico. Infancia y Aprendizaje, 42, pp. 37-63.

PFUNDT, H. y DUIT, R. (1993). Bibliography: Students' alternative frameworks and science education. Kiel: University of Kiel.

PORLÁN, R. (1986). Más allá de la investigación del medio Cuadernos de Pedagogía, 142, pp. 8-12.

PORLÁN, R. (1989). Teoría del conocimiento, teoría de la enseñanza y desarrollo profesional. Las concepciones epistemológicas de los profesores. Tesis doctoral. (Versión en microficha núm. 9. Sevilla: Editorial de la Universidad de Sevilla, 1992).

PORLÁN, R. (1993a). La didáctica de las ciencias. Una disciplina emergente. Cuadernos de Pedagogía, 210, pp. 68-71.

PORLÁN, R. (1993b). Constructivismo y escuela. Hacia un modelo de enseñanza-aprendizaje basado en la investigación. Sevilla: Díada Editora.

PORLÁN, R. (1993c). La identidad epistemológica de la didáctica de las ciencias experimentales, en Montero, L. y Vez, J.M. (eds.): La didácticas específicas en la formación del profesorado. Santiago de Compostela: Tórculo Edicións.
PORLÁN, R. y MARTÍN, J. (1994). EI saber práctico de los profesores especialistas. Aportaciones desde las didácticas específicas. Investigación en la Escuela, 24, pp. 49-59. (Trad. francesa, Le savoir practique des enseignants spécialisés. Apports des didactiques spécifiques. Aster, 19 , pp. 49-60, 1994)

SAN MARTÍN, A. (1984). Estudio de los procesos de pensamiento del profesor en formación. Tesis doctoral inédita. Universitat de València.

SHAVELSON, R. y STERN, P. (1981). Research on teachers' pedagogical thoughts, judgements, decisions and behaviour. Review of Educational Research, 51, 4, pp. 445-498.

SCHIBECI, R.A. (1986). Images for science and scientists and science education. Science Education, 70 (2), pp. 139-149.

SHULMAN, L.S. (1981). Recent developinents in the study of teaching, en Tabachnick, B.R., Popkewtiz, T.S. y Szekely, B.B. (eds.). Teacher thinking: a new perspective on persisting problems in education. Lisse: Swets and Zeitlinger.

TIBERGHIEN, A. (1985). Quelques éléments sur l'evolution de la recherche en didactique de la physique. Revue Française de Pédagogie, 72, pp. 71-86.

TOULMIN, S. (1972). Human Understanding. Vol. I: The Collective Use and Evolution of Concepts. Princeton: Princeton University Press. (Trad. cast. La comprensión humana. Vol. I: El uso colectivo y la evolución de los conceptos. Madrid: Alianza, (977).

VEGA, M. (1984). Introducción a la psicología cognitiva. Madrid: Alianza.

YINGER, R.J. y CLARK, C.M. (1982). Understanding teachers' judgements about instruction: the task, the method and the meaning. Research Series, 121. East Lansing. Michigan. 